\section{Continuous positive airway pressure in congenital lobar emphysema}

Congenital lobar emphysema with air trapping distal to defective bronchial cartilage is an unusual cause of respiratory distress in early infancy. ${ }^{1}$ Prompt treatment, usually lobectomy, may be necessary to maintain ventilation. Until recently medical management had little to offer; indeed, mortality was likely to be increased by this approach. ${ }^{2}$ We describe an infant for whom a period of medical management as a preliminary to definitive surgery was feasible through maintaining ventilation by continuous positive airway pressure (CPAP).

\section{Case report}

In a 4330 -g baby girl severe respiratory distress was present from birth, but expiratory obstruction with over-distension of the right middle and left upper lobes was apparent only after several weeks. To prevent episodes of respiratory failure before surgery, CPAP was maintained through a nasotracheal tube for 23 weeks. After removal of the grossly emphysematous left upper and right middle lobes she was extubated without difficulty.

The East-Radcliffe circuit with "dummy lung," as used for the ventilation of neonates, ${ }^{3}$ was further modified for spontaneous respiration with CPAP by connecting the expired air-port to an underwater blow-off adjusted to the desired pressure, in this case $4-6 \mathrm{~cm}$ of water (figure). The ventilator

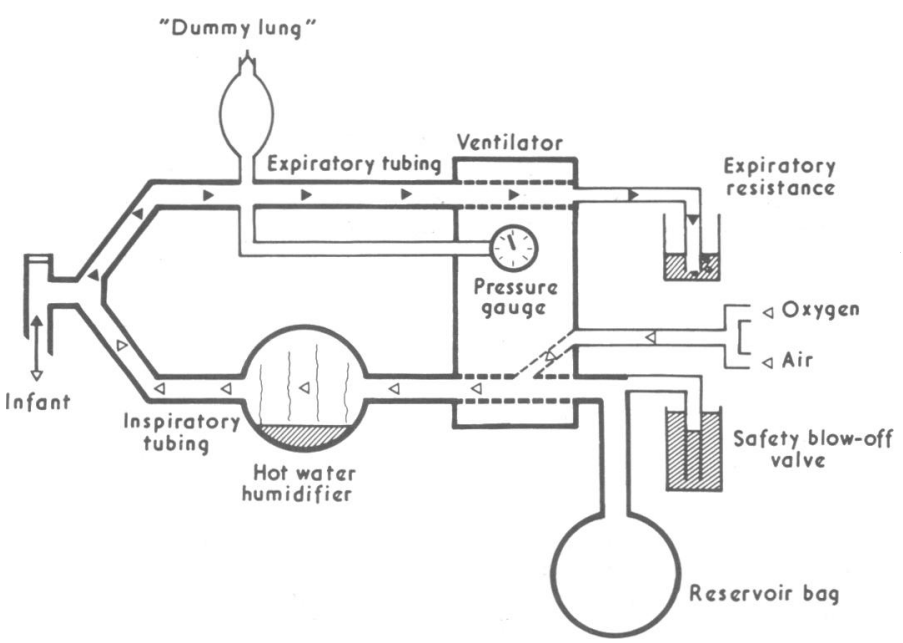

The East-Radcliffe ventilatory circuit modified for continuous positive airway pressure (CPAP) during spontaneous respiration.

switch was placed in the "bag" position and the required oxygen/air mixture was delivered into the oxygen port. A total gas flow of 5 litres per minute was adequate to prevent rebreathing and to maintain an adequate temperature of the humidified gases at the endotracheal tube connector. The air intake port was connected to the reservoir bag, whose blow-off valve was closed, and an additional $10 \mathrm{~cm}$ underwater blow-off inserted at this point to prevent excessive pressure should obstruction occur on the expiratory limb. In this way the manometer and the humidifier of the respirator could be used during spontaneous respiration. The reservoir bag gave additional visual indication of the pressure in the circuit and a ready means of producing an artificial sigh or manual ventilation. Intermittent positive-pressure ventilation could be instituted by pushing the ventilator switch to the "pump" position.

With Portex ivory vinyl nasal tubes the Tunstall modification of the new Oxford pattern endotracheal tube connector* was used. This was further modified to ensure that the metal part of the connector was held clear of the nose-an important point of detail in preventing erosion of the nares during prolonged nasotracheal intubation. Part of a wooden tongue depressor was strapped to the wire frame longitudinally. This was held by tape running over the head in the midline and, by using a piece of foam rubber on the lower forehead as a fulcrum, this effectively levered the endotracheal tube connector out against the pull of the anchoring lower straps.

\section{Comment}

Abnormalities that prevent normal deflation of the lung or of individual lobes include a wide variety of conditions that defy simple

*Penlon Ltd, Abingdon, Oxford. nomenclature or classification but they all operate by creating a check-valve effect in the bronchial lumen. ${ }^{4}$ An abnormality of bronchial cartilage is probably the most important factor in aetiology. ${ }^{5}$

Once the diagnosis is suspected bronchoscopy is desirable to exclude obstruction by aspirated material or from external compression. In the severely distressed infant it is usually advisable to proceed to lobectomy, especially when only one lobe is affected. Clarification of the lobe or lobes affected may be difficult, or surgery may not be immediately available, and then it is reasonable to postpone operation until the optimum time, provided that ventilation can be safeguarded. For this purpose a continuous inflating pressure seems logical, as by its splinting action airway patency can be maintained to allow the infant to breathe spontaneously. CPAP is now a familiar technique in most newborn nurseries, and, as this report indicates, it is feasible in older infants for long periods without serious sequelae.

1 Urban, A E, Stark, J, and Waterston, D J, Thoraxchirurgie, 1975, 23, 255.

2 Leape, L L, and Longino, L A, Pediatrics, 1964, 34, 246.

3 Tunstall, M E, et al, Archives of Disease in Childhood, 1968, 43, 486.

4 Thomson, J, and Forfar, J O, Archives of Disease in Childhood, 1958, 33, 97.

${ }^{5}$ Campbell, P E, Australian Paediatric fournal, 1969, 5, 226.

(Accepted 7 February 1977)

Departments of Child Health and Anaesthetics, Royal Aberdeen

Children's Hospital and University of Aberdeen

A G M CAMPBELL, MB, FRCP, professor of child health

$M$ E TUNSTALL, MB, FFARCS, consultant anaesthetist

A J SHEARER, MB, FFARCS, registrar in anaesthetics

\section{Conception of normal child during chemotherapy of acute lymphoblastic leukaemia in the father}

There are few and conflicting data about the offspring of male patients treated with cytotoxic agents. ${ }^{12}$ We report the outcome of two pregnancies in the wife of a young man receiving chemotherapy for acute lymphoblastic leukaemia (ALL).

\section{Case history}

ALL was diagnosed in a 18-year-old man in March 1973. Complete remission was obtained after four weeks' treatment with vincristine ( $2 \mathrm{mg}$ weekly) and prednisone ( $75 \mathrm{mg}$ daily) followed by a 10-day course of $\mathrm{L}$ asparaginase. Prophylactic treatment to the central nervous system with irradiation of the skull and six intrathecal injections of methotrexate (MTX) was given. Maintenance chemotherapy was started in May 1973 with oral 6-mercaptopurine (6-MP) (100-150 mg daily) and oral MTX (25 mg once a week). Every three months two-week reinduction courses with vincristine and prednisone were given in addition.

Against our advice the patient and his wife decided to have children while he was still under chemotherapy. A first pregnancy ended in the third month with a spontaneous abortion in June 1974. No fetal tissue could be obtained for chromosomal studies. A second uneventful pregnancy ended after 35 weeks' gestation on 27 September 1975 with the birth of a healthy premature girl with appropriate birth weight $(2360 \mathrm{~g})$ and without detectable malformations. Chromosomal studies of peripheral blood lymphocytes of the child showed no abnormalities.

The fatherhood of our patient has been proved by appropriate forensic serological testing. There is no reason to suppose that the patient failed to take continuously the prescribed drugs.

\section{Discussion}

There are well-known effects of cytotoxic agents to the reproductive system. They can impair testicular and ovarian function, leading eventually to infertility, and they may be teratogenic when given to the mother during the first trimester of pregnancy. ${ }^{3}$ Little is known, however, about an eventual mutagenic effect on spermatogenesis. The rapid cell turnover of spermatogenic cells could predispose them 